\title{
Comparative evaluation of epidural bupivacaine alone and bupivacaine combined with magnesium sulfate in providing postoperative analgesia: a meta-analysis of randomized controlled trials
}

\section{Li-Qin Li}

Guizhou Provincial People's Hospital

Hou-Zhong Zhang ( $\sim$ 2994266687@qq.com)

Jilin University Second Hospital https://orcid.org/0000-0002-3438-4781

\section{Mei-Dan Fang}

Jilin University Second Hospital

\section{Cong Wang}

First Affiliated Hospital to Changchun University of Chinese Medicine

Hong-Liu Lu

Jilin University Second Hospital

\section{Li-Xue Wang}

Jilin University Second Hospital

Hong-Yu Xu

Jilin University Second Hospital

\section{Research article}

Keywords: Magnesium sulfate, Postoperative analgesia, Epidural anesthesia, Bupivacaine

Posted Date: January 9th, 2020

DOI: https://doi.org/10.21203/rs.2.17939/v2

License: (c) (7) This work is licensed under a Creative Commons Attribution 4.0 International License. Read Full License

Version of Record: A version of this preprint was published at BMC Anesthesiology on February 5th, 2020. See the published version at https://doi.org/10.1186/s12871-020-0947-8. 


\section{Abstract}

Background The comparative efficacy of epidural bupivacaine alone and bupivacaine combined with magnesium sulfate in providing postoperative analgesia remains controversial.Methods We searched Mediline (OvidSP), EMBASE (OvidSP) and Cochrane Central Register of Controlled Trials (CENTRAL) to identify trials that compared epidural bupivacaine and magnesium sulfate combination (intervention) with bupivacaine alone (control). Grading of Recommendations, Assessment, Development and Evaluations (GRADE) framework was used to assess the quality of evidence.Results Eleven studies fulfilled our inclusion criteria after screening. We found that epidural bupivacaine combined with magnesium sulfate could prolong the time for first rescue analgesics (SMD 4.96; 95\% Cl [2.75, 7.17], P<0.00001, I 2 = $98 \%$ ), reduce the number of patients who need rescue analgesics (RR $0.38 ; 95 \% \mathrm{Cl}[0.20,0.74], \mathrm{P}=0.004, \mathrm{I} 2=75 \%$ ) and requirement for rescue analgesics (SMD -2.65; 95\% Cl [-4.23, -1.06], P=0.001, I 2 = 96\%).Conclusions Magnesium suifate as an adjuvant of epidural bupivacaine improved postoperative analgesia. However, we rated the quality of evidence to be very low because of high heterogeneity, imprecise of results and small sample sizes. Furthermore, further large highquality trials are still needed to confirm the effects of magnesium sulfate on postoperative analgesia.

\section{Background}

Alleviation of postoperative pain is an important objective for the anesthesiologists. Inadequaten postoperative pain control is associated with deep vein thrombosis and pulmonary embolism, quality-of-life impairment, delayed recovery time and higher health-care costs [1,2]. Epidural anaesthesia is an effective technique, with the advantage of safety, efficiency and prolonged postoperative pain relief [3]. A number of adjuvants have been used with bupivacaine via epidural route over the years with the purpose of prolonging the duration of postoperative analgesia and minimising the side effects. Nevertheless researchers continue to find the optimum adjuvants, as the currently researched adjuvants (e.g. opioids, tramadol, dexmedetomidine) still have some adverse effects such as nausea and vomiting, pruritus, bradycardia, and hypotension [4-6].

Magnesium is the fourth most plentiful cation in the body and possess certain analgesic property in both animal and human models of pain $[7,8]$. Magnesium ion $(\mathrm{Mg} 2+)$ is a non-competitive N-methyl-D-aspartate (NMDA) receptor antagonist that blocks inward current flow through ion channels linked to NMDA receptors in a voltage-dependent fashion, has the potential to prevent central sensitization induced by peripheral nociceptive stimulation. For these reasons, it seems plausible that magnesium as an adjuvant of epidural bupivacaine can prolong postoperative analgesia and reduce side effects. Therefore we conducted this meta-analysis to test our hypothesis.

\section{Materials And Methods}

For this meta-analysis, we followed the recommendation with Preferred Reporting Items for Systematic Reviews and Meta-Analyses (PRISMA) guidelines [10], and the quality of the evidence was assessed using the GRADE approach and recommendations from the Cochrane Collaboration [11].

\subsection{Eligibility and exclusion criteria}

The purpose of this meta-analysis was to evaluate the safety and efficacy of magnesium sulfate as an adjuvant of epidural bupivacaine. Studies will be included if they meet the following criteria: (1) randomized controlled trials (RCTs); (2) adults ( $\geq 18$ years old); (3)comparing the analgesic efficacy of epidural bupivacaine and magnesium sulfate combination (intervention), with bupivacaine alone (control) for anesthesia or postoperative pain management; (4) study provided data at least on one of the outcomes (time to the first rescue analgesia, number of patients required rescue 
analgesia, requirement for rescue analgesia, duration of motor block, and side effects); (5) full text published in English. We excluded studies in which another drug (eg.fentanyl, morphine) was added in the intervention or control group, and magnesium sulfate was administered by another route (e.g. intrathecal, intravenous or intramuscular).

\subsection{Endpoints}

Primary outcomes: (1) the time to the first request for rescue analgesics; (2) the number of patients required postoperative rescue analgesics; (3) requirement for rescue analgesics. Secondary outcomes: (1) duration of motor block; (2) adverse events related to postoperative analgesia protocols (the incidence of hypotension, bradycardia, nausea and vomiting, pruritus, shivering).

\subsection{Search strategy and study selection}

We searched Mediline (OvidSP), EMBASE (OvidSP) and Cochrane Central Register of Controlled Trials (CENTRAL) on october 24, 2019 in order to identify trials that compared epidural bupivacaine and magnesium sulfate combination (intervention) with bupivacaine alone (control). The exact search strategies are shown in Appendix 1. After importing the search results into EndNote X9, duplicated studies were excluded. Two investigators (Li and Wang) independently determined eligibility on the basis of the title, abstract and full text according to the inclusion and exclusion criteria, with disagreements resolved by discussion and consensus with a third agent (Fang).

\subsection{Data extraction and quality assessment}

Two review authors ( $\mathrm{Li}$ and Wang), independently extracted data using a predesigned form and verified for consensus before entry into Review Manager 5.0. Li and Wang independently assessed the risk of bias for each included study using the criteria outlined in the Cochrane Handbook for Systematic Reviews of Interventions [12]. We rated the overall risk of bias of a study as low if $\leqq 1$ domain were 'high risk' or 'unclear', high if two or more of the domains were identified as 'high risk' or 'unclear'. Resolving any disagreements by discussion and consensus with a third reviewer (Fang).

\subsection{Statistical analysis}

We used the software Review Manager 5.0 for statistical analysis. Only primary and secondary outcomes defined previously were included in our analysis. For continuous data (e.g. the time to the first request for rescue analgesics, requirement for rescue analgesics and duration of motor block), considering the different modes of postoperative pain management and species of rescue analgesics, we calculated standardized mean differences (SMDs) with corresponding $95 \%$ confidence intervals $(95 \% \mathrm{Cl})$. We rated the effect size of SMDs as small effect $(0.2-0.5)$, medium effect (0.5-0.8) and large effect $(\geq 0.8)$. For those studies that did not report a mean and standard deviation (SD), we did not hand and transformate the date, because we did not know if it was normally distributed. We calculated risk ratios (RR) with corresponding $95 \% \mathrm{Cl}$ for dichotomous outcomes (e.g. the number of patients required postoperative rescue analgesics, adverse events related to postoperative analgesia protocols).

\subsection{Assessment of heterogeneity and subgroup analysis}


We assessed statistical heterogeneity using the $\mathrm{I}^{2}$ statistic, as described in the Cochrane Handbook for Systematic Reviews of Interventions [12], and an $I^{2}$ value $>50 \%$ is considered to indicate substantial heterogeneity. To explore the sources of clinical heterogeneity, we planned to perform subgroup analyses of bolus injection versus bolus followed a continuous injection according to the administration of magnesium sulfate. We used a randomized-effect model if there was significant heterogeneity among studies $\left(I^{2}>50 \%\right)$, otherwise the fixed effects model was used. A P-value $<0.05$ and the $95 \% \mathrm{Cl}$ did not cross the equivalent line were considered statistically significant differences from control.

\subsection{Sensitivity analysis and assessment of publication bias}

We decided to perform sensitivity analyses for the primary outcomes by removing studies with high risk of of bias or using two different models (the randomized effect model and the fixed effect model). If sufficient studies (10 or more) were included for the primary or second outcomes, we had intended to use a funnel plot to explore the possibility of publication bias.

\section{Results}

\subsection{Study selection and characteristics of included studies}

The literature search identified 4392 studies totally and 112 references from CENTRAL, 2481 references from MEDLINE (OvidSP), and 1799 studies from Embase (Ovidsp). Eleven studies fulfilled our inclusion criteria after screening [13-23]. The details of retrieval was shown in figure. 1. The various characteristics of the included studies were shown in Table 1. Seven trials applied magnesium sulfate as a bolus followed by a continuous infusion [13-19], while the remaining four trials applied a bolus injection [20-23]. In eight studies enrolled participants undergoing Lower abdominal or lower limb surgeries [13,14,16,18-21,23], with participants administered epidural anesthesia. Participants in Mohammad 2015 and Radwan 2017 underwent unilateral thoracic surgery and spine surgery respectively with general plus epidural anesthesia. Two studies enrolled participants undergoing cesarean section, with participants in Sun 2012 administered combines spinal epidural anesthesia, and participants in Elsharkawy 2018 administered epidural anesthesia. Total enrollment ranged from 40 to 100 participants, with the number of participants in each study epidural bupivacaine and magnesium sulfate combination ranging from 20 to 50 , control 20 to 50 .

\subsection{Risk of Bias of the included trials}

Figure. 2 and Figure. 3 presents the risk of bias of the included studies. We assessed 3 of these trials as low risk of bias $[13,14,17]$, while the remaining 8 trials as high risk of bias according to our pre-specified criteria $[15,16,18-23]$.

\subsection{Primary outcome: the time to the first request for rescue analgesics}

Nine studies reported the time to the first request for rescue analgesics, in which the data of six studies were reported as mean \pm standard deviation (SD) $[13-16,18,21]$, while the remaining two studies reported the data in figure $[17,20,23]$. As a result, only six studies involving 400 patients were included for analysis [13-16,18,21]. The meta-analysis showed that the time to the first request for rescue analgesics was prolonged significantly in the magnesium sulfate group compared with the control group (SMD 4.96; 95\% Cl [2.75, 7.17], $\mathrm{P}<0.00001, \mathrm{I}^{2}=98 \%$; Figure. 4). We deemed the quality of the evidence to be very low because: (1) four studies had 'high' risk of bias; (2) the result was imprecise (wide confidence interval); and (3) there was significant heterogeneity among studies. 
Subgroup analysis was conducted according to the administration of magnesium sulfate. Five studies administrated magnesium sulfate by bolus injection [13-16,18], and only one study by bolus followed a continuous injection [21]. We noted the time to the first request for rescue analgesics was also prolonged significantly in the magnesium sulfate group compared with the control group when the study by bolus followed a continuous injection was excluded (SMD 3.67; 95\% $\left.\mathrm{Cl}[1.75,5.58], \mathrm{P}=0.0002, \mathrm{I}^{2}=97 \%\right)$. We also found significant difference between the subgroups $(\mathrm{P}<0.0001)$ and the bolus followed a continuous injection subgroup had a greater effect on the time to the first rescue analgesics.

\subsection{Primary outcome: the number of patients required postoperative rescue analgesics}

Five studies including 304 patients reported the number of patients required rescue analgesics $[13,17,19,20,22]$. The merged effect analysis showed that it was significantly less in magnesium group compared with control group (RR 0.38; $95 \% \mathrm{Cl}[0.20,0.74], \mathrm{P}=0.004, \mathrm{I}^{2}=75 \%$, Figure. 5 ). We judged, the quality of the evidence to be very low based on the GRADE framework: (1) three studies had a 'high' risk of bias; (2) there was significant heterogeneity among studies.

Subgroup analysis was conducted according to the administration of magnesium sulfate. Patients required postoperative rescue analgesics was significantly less in magnesium group when magnesium sulfate was administrated by bolus injection (RR 0.49; 95\% Cl [0.24, 0.97], P = 0.04, $1^{2}=70 \%$ ). No significant difference was shown between the two groups when magnesium sulfate was administrated by bolus followed a continuous injection (RR $0.17 ; 95 \% \mathrm{Cl}[0.01,1.97], \mathrm{P}=$ $\left.0.16,1^{2}=84 \%\right)$

\subsection{Primary outcome: requirement for rescue analgesics}

The requirement for rescue analgesia was reported in five studies involving 300 participants $[13,15,18,20,23]$. The metaanalysis showed that the magnesium sulfate group had significantly lower consumption of rescue analgesics than the control group (SMD -2.65; 95\% Cl [-4.23,-1.06], $\mathrm{P}=0.001, \mathrm{I}^{2}=96 \%$; Figure. 6). We rated the quality of the evidence to be very low: (1) four studies had 'high' risk of bias; (2) the result was imprecise; and (3) there was significant heterogeneity among studies.

Subgroup analysis was conducted according to the administration of magnesium sulfate. A significant reduction was shown in the magnesium group when magnesium sulfate was administrated by bolus injection (SMD $-2.92 ; 95 \% \mathrm{Cl}[-5.43$, -0.42], $P=0.02, I^{2}=97 \%$ ). No significant difference was shown between two groups when magnesium sulfate was administrated by bolus followed a continuous injection (SMD $-2.31 ; 95 \% \mathrm{Cl}[-5.26,0.65], \mathrm{P}=0.13, \mathrm{I}^{2}=98 \%$ ).

\subsection{Secondary outcome: duration of motor block}

Two studies reported duration of motor block $[13,16]$. The result showed that the duration of motor block in magnesium sulfate group was significantly longer than contol group (SMD 6.29; 95\% $\mathrm{Cl}[0.33,12.24], \mathrm{P}=0.04, \mathrm{I}^{2}=97 \%$; Figure. 7). We rated the quality of the evidence to be very low: (1) the result was imprecise; (2) there was significant heterogeneity among studies; and (3) limited availability of evidence.

\subsection{Secondary outcome: adverse events related to postoperative analgesia protocols}


A meta-analysis of adverse events was shown in Figure. 8, such as hypotension ( $\mathrm{RR} 0.80 ; 95 \% \mathrm{Cl}[0.64,1.00], \mathrm{P}=0.05$, $\mathrm{I}^{2}=0 \%$ ), bradycardia (RR 0.80; $95 \% \mathrm{Cl}[0.47,1.36], \mathrm{P}=0.41, \mathrm{I}^{2}=0 \%$ ), nausea and vomiting ( $\mathrm{RR} 0.57 ; 95 \% \mathrm{Cl}[0.31,1.06], \mathrm{P}=$ $0.08, \mathrm{I}^{2}=0 \%$ ) and pruritus (RR $0.67 ; 95 \% \mathrm{Cl}[0.11,3.91], \mathrm{P}=0.65, \mathrm{I}^{2}=0 \%$ ) showed no statistically significant differences, except that the incidence of shivering in magnesium sulfate group was significantly lower than control group (RR 0.31; $\left.95 \% \mathrm{Cl}[0.18,0.53], \mathrm{P}<0.0001, \mathrm{I}^{2}=24 \%\right)$. We rated the evidence for adverse events to be moderate quality.

\subsection{Sensitivity analysis and assessment of publication bias}

Based on the prior definition, there were only three studies with a low risk of bias $[13,14,17]$, so we did not conduct the sensitivity analysis based on the risk of bias. Sensitivity analyses of primary outcomes using the fixed effect model yielded stable overall results. Consider that each outcome included fewer than 10 studies, there were insufficient data for any publication bias analysis.

\section{Discussion}

This meta-analysis aimed to assess the efficacy and safety of magnesium sulfate as an adjuvant in epidural bupivacaine in providing postoperative analgesia. We found that epidural bupivacaine combined with magnesium sulfate could prolong the time for first rescue analgesics. Furthermore, the addition of magnesium sulfate could reduce the number of patients who need rescue analgesics and requirement for rescue analgesics without adverse events. In addition, our metaanalysis showed that the incidence of shivering was lower with bupivacaine-magnesium sulfate than bupivacaine alone. Nevertheless, duration of motor blockade was significantly prolonged in group magnesium sulfate.

We found that many studies did not adequately report randomization methods. Five studies did not describe the generation of random sequences in detail $[13,15,16,20,23]$, two studies did not describe the methods of allocation concealment $[18,19]$, three studies did not describe whether the anaesthetists were blind for this study design $[15,16,21]$ and four studies did not report in detail their blind assessment of the outcomes [15,16,21,22]. Only one studies reported clinical trials registration [13] and we were not clear about the risk of selective outcome reporting bias. We judged evidence for the time to the first request for rescue analgesics, the number of patients required rescue analgesics, requirement for rescue analgesia and duration of motor block to be very low certainty, and evidence for adverse events to be moderate quality. The low quality of evidence for primary outcomes was largely due to significant heterogeneity among studies and imprecision of the result. The significant heterogeneity might be explained by the differences in the types of surgery performed, the doses and manners of magnesium sulfate administered, and the postoperative pain management models. However, results of subgroup analyses on manners of magnesium administered did not appear to explain heterogeneity and we found that high levels of statistical heterogeneity still remained in both subgroups in each analysis. The surgery types varied between studies to include: cesarean section, unilateral thoracic, spinal, abdominal, orthopaedic. These differences may contribute to inconsistency and reduce the overall applicability of the evidence.

Whether the administrative route is intravenous, epidural, or intrathecal, the actual site of action of magnesium sulfate is probably at the spinal cord NMDA receptors. The analgesic effect primarily depends on $\mathrm{Mg} 2+$ blocks inward current flow through ion channels linked to NMDA receptors [9]. Although a previous systematic review found that intravenous administration of magnesium sulfate in orthopedic surgery could reduce postoperative requirement for postoperative analgesics and adverse events such as vomiting, nausea, and shivering [24], the role of intravenous magnesium sulfate was controversial. Some studies had found that intravenous magnesium failed to improve postoperative pain in gastrointestinal surgery [25] and in a pediatric population undergoing tonsillectomy [26]. The reason may be the limited ability of magnesium ions to penetrate the blood-brain barrier [27], so it seems plausible that epidural or intrathecia magnesium might be more effective. An earlier meta-analysis focused on cesarean section revealed that the additional 
neuraxial magnesium sulfate exerted significant effects on prolonging the duration of neuraxial anesthesia, reducing postoperative pain scores and decreasing requirement for postoperative analgesics, and was therefore in broad similar to our findings [28]. Furthermore, our findings are similar with the findings in a recent published meta-analysis, where the authors reported a reduction of the need for postoperative rescue analgesics in pediatric when magnesium was added to local anesthetics for caudal anesthesia [29].

In addition, magnesium sulfate has been compared with other epidural adjunct analgesic drugs. One study that evaluated the effects of epidural magnesium sulfate versus dexmedetomidine, and found that the time from epidural medication to first rescue analgesics was longer in dexmedetomidine group, duration of sensory and motor blockade was significantly prolonged in group dexmedetomidine, but risk of sedation increased and there was fall in the mean pulse rate in group dexmedetomidine [16]. Radwan et al. compared magnesium sulfate with fentanyl [22] and Mohammad et al. compared magnesium sulfate with clonidine [15] found the effect of magnesium sulfate on postoperative pain to be comparable to that of the other drugs.

In this meta-analysis, the addition of magnesium sulfate significantly prolonged the duration of motor block. The mechanism might be that magnesium inhibits the motor endplate release of acetylcholine due to inhibition of calciumdependent channels $[30,31]$. As for adverse effects, our meta-analysis showed that the incidence of shivering was lower in magnesium sulfate group. The reason might be that perioperative magnesium supplementation prevented the postoperative hypomagnesaemia and decreased the incidence of postoperative shivering [32].

There are some limitations to this study. Firstly, our meta-analysis demonstrates efficacy of epidural bupivacaine combined with magnesium sulfate when compared with bupivacaine alone in providing postoperative analgesia. Even if It is possible that our findings cannot be interpreted as truly positive because of the small sample sizes and the low quality of evidence assessed by GRADE framework; Secondly, We found that many studies did not adequately report randomization methods; Third, we didn't assess publication bias due to the limited number of studies; Fourth, our findings showed high heterogeneity among studies, especially the existing clinical heterogeneity, such as types of surgery performed, the doses and manners of magnesium sulfate administered, and the postoperative pain management models.

\section{Conclusion}

In conclusion, This meta-analysis revealed that magnesium suifate as an adjuvant of epidural bupivacaine improves postoperative analgesia. However, we rated the quality of evidence to be very low because of high heterogeneity, imprecise of results and small sample sizes. Furthermore, further large high-quality trials are still needed to confirm the effects of magnesium sulfate on postoperative analgesia.

\section{Abbreviations}

NMDA: N-methyl-D-aspartate, PRISMA: Preferred Reporting Items for Systematic Reviews and Meta-Analyses, RCTs: randomized controlled trials, SMDs: standardized mean differences, $\mathrm{Cl}$ : confidence intervals, RR: relative risk, SD: standard deviation.

\section{Declarations}

\section{Acknowledgements}

Not applicable.

\section{Funding}


There was no funding source in this study.

Availability of data and material

All data generated or analysed during this study are included in this published article.

\section{Authors' contributions}

LLQ participated in the design, collected the data, performed the quality assessment. ZHZ participated in the design and draft the manuscript. WC and FMD collected the data, performed the quality assessment, and helped to draft the manuscript. XHY, LHL, WLX helped to perform statistical analyses and search strategy. All authors read and approved the final manuscript.

\section{Ethics approval and consent to participate}

Not applicable.

\section{Consent for publication}

Not applicable.

\section{Competing interests}

The authors declare that they have no competing interests.

\section{Reference}

1. Gan TJ. Poorly controlled postoperative pain: prevalence, consequences, and prevention. J Pain Res. 2017; 10:228798.

2. Joshi GP, Ogunnaike BO. Consequences of inadequate postoperative pain relief and chronic persistent postoperative pain. Anesthesiol Clin North Am. 2005; 23(1):21-36.

3. $\mathrm{Ng} \mathrm{A}$, Swanevelder J. Pain relief after thoracotomy: is epidural analgesia the optimal technique? Br J Anaesth. 2007; 98(2):159-62.

4. Tan X, Shen L, Wang L, et al. Incidence and risk factors for epidural morphine induced pruritus in parturients receiving cesarean section: A prospective multicenter observational study. Medicine (Baltimore). 2019; 98(40):e17366.

5. Turkoglu Z, Karacaer F, Biricik E, et al. Comparison of the Effects of Epidural Levobupivacaine with Tramadol or Morphine Addition on Postoperative Analgesia following Major Abdominal Surgery. Turk J Anaesthesiol Reanim. 2019; 47(4):287-94.

6. Sekhar DP, Kumar L, Kesavan R, et al. Comparison of the Analgesic Efficacy of a Single Dose of Epidural Dexmedetomidine versus Fentanyl as an Adjuvant to Bupivacaine in Abdominal Surgery. Anesth Essays Res . 2019; 13(3):465-70.

7. Begon S, Pickering G, Eschalier A, et al. Magnesium increases morphine analgesic effect in different experimental models of pain. Anesthesiology. 2002; 96(3):627-32.

8. Kogler $\mathrm{J}$. The analgesic effect of magnesium sulfate in patients undergoing thoracotomy. Acta Clin Croat. 2009; 48(1):19-26.

9. Mayer ML, Westbrook GL, Guthrie PB. Voltage-dependent block by Mg2+ of NMDA responses in spinal cord neurones. Nature. 1984; 309(5965):261-3. 
10. Liberati A, Altman DG, Tetzlaff J,et al. The PRISMA statement for reporting systematic reviews and meta-analyses of studies that evaluate healthcare interventions: explanation and elaboration. BMJ. 2009; 339:b2700.

11. Guyatt GH, Oxman AD, Vist GE,et al. GRADE: an emerging consensus on rating quality of evidence and strength of recommendations. BMJ. 2008; 336(7650):924-6.

12. Higgins JPT, Green S (editors). Cochrane Handbook for Systematic Reviews of Interventions Version 5.1.0 [updated March 2011]. The Cochrane Collaboration. 2011. Available from http://www.cochrane-handbook.org.

13. Elsharkawy RA, Farahat TE, Abdelhafez MS. Analgesic effect of adding magnesium sulfate to epidural levobupivacaine in patients with pre-eclampsia undergoing elective cesarean section. J Anaesthesiol Clin Pharmacol. 2018; 34(3):328-34.

14. Ghatak T, Chandra G, Malik A et al. Evaluation of the effect of magnesium sulphate vs. clonidine as adjunct to epidural bupivacaine. Indian J Anaesth. 2010; 54(4):308-13.

15. Mohammad W, Mir SA, Mohammad K, et al. A randomized double-blind study to evaluate efficacy and safety of epidural magnesium sulfate and clonidine as adjuvants to bupivacaine for postthoracotomy pain relief. Anesth Essays Res. 2015; 9(1):15-20.

16. Shahi V, Verma AK, Agarwal A, et al. A comparative study of magnesium sulfate vs dexmedetomidine as an adjunct to epidural bupivacaine. J Anaesthesiol Clin Pharmacol. 2014; 30(4):538-42.

17. Sun J, Wu X, Xu X, et al. A comparison of epidural magnesium and/or morphine with bupivacaine for postoperative analgesia after cesarean section. Int J Obstet Anesth. 2012; 21(4):310-6.

18. Gupta A, Goyal VK, Gupta N, et al. A randomized controlled trial to evaluate the effect of addition of a single dose of epidural magnesium sulphate on the duration of postoperative analgesia in patients undergoing lower abdominal surgeries under epidural anaesthesia. Sri Lankan Journal of Anaesthesiology. 2013; 21(1):27-31.

19. Shruthi AH, Sudheesh K, Nethra SS, et al. The Effect of a Single Dose of Magnesium Sulphate as an Adjuvant to Epidural Bupivacaine for Infraumbilical Surgeries: A Prospective Double-Blind, Randomized Control Trial. Middle East Journal of Anesthesiology. 2016; 23(4):449-55.

20. Aly Kandil AH, Hammad RAEA, Shafei MAE, et al. Preemptive use of epidural magnesium sulphate to reduce narcotic requirements in orthopedic surgery. Egyptian Journal of Anaesthesia. 2011; 28(1):17-22.

21. Omar H. Magnesium Sulfate as a Preemptive Adjuvant to Levobupivacaine for Postoperative Analgesia in Lower Abdominal and Pelvic Surgeries under Epidural Anesthesia(Randomized Controlled Trial). Anesth Essays Res. 2018; 12(1):256-61.

22. Radwan T, Awad M, Fahmy R, et al. Evaluation of analgesia by epidural magnesium sulphate versus fentanyl as adjuvant to levobupivacaine in geriatric spine surgeries. Randomized controlled study. Egyptian Journal of Anaesthesia. 2017; 33(4):357-63.

23. Daabiss MA, Kandil A. Evaluation of the effect of magnesium vs. midazolam as adjunct to epidural bupivacaine in patients undergoing total knee replacement. British Journal of Medical Practitioners. 2013; 6(2):a610.

24. Peng YN, Sung FC, Huang ML, et al. The use of intravenous magnesium sulfate on postoperative analgesia in orthopedic surgery: A systematic review of randomized controlled trials. Medicine (Baltimore). 2018; 97(50):e13583.

25. Guo BL, Lin Y, Hu W, et al. Effects of Systemic Magnesium on Post-operative Analgesia: Is the Current Evidence Strong Enough? Pain Physician. 2015; 18(5):405-18.

26. Xie M, Li XK, Peng Y. Magnesium sulfate for postoperative complications in children undergoing tonsillectomies: a systematic review and meta-analysis. J Evid Based Med. 2017; 10(1):16-25.

27. Ko SH, Lim HR, Kim DC,et al. Magnesium sulfate does not reduce postoperative analgesic requirements. Anesthesiology. 2001; 95(3):640-6. 
28. Wang SC, Pan PT, Chiu HY, et al. Neuraxial magnesium sulfate improves postoperative analgesia in Cesarean section delivery women: A meta-analysis of randomized controlled trials. Asian J Anesthesiol. 2017; 55(3):56-67.

29. Kawakami H, Mihara T, Nakamura N, et al. Effect of magnesium added to local anesthetics for caudal anesthesia on postoperative pain in pediatric surgical patients: A systematic review and meta-analysis with Trial Sequential Analysis. PLoS One. 2018; 13(1):e0190354.

30. Ghoneim MM, Long JP. The interaction between magnesium and other neuromuscular blocking agents. Anesthesiolog 1970; 32(1):23-7.

31. Kim $\mathrm{MH}$, Oh AY, Jeon $\mathrm{YT}$, et al. A randomised controlled trial comparing rocuronium priming, magnesium pretreatment and a combination of the two methods. Anaesthesia. 2012; 67(7):748-54.

32. Lysakowski C, Dumont L, Czarnetzki C, et al. Magnesium as an adjuvant to postoperative analgesia: a systematic review of randomized trials. Anesth Analg. 2007; 104(6):1532-9.

\section{Table}

\section{Table 1 Characteristics of included trials}




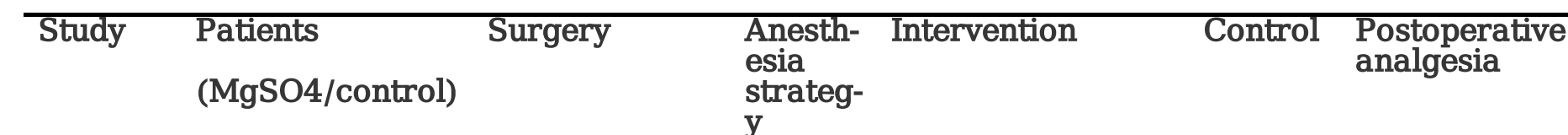

Aly 30/30

2011

Orthoped-ic

Daabiss $\quad 40 / 40$

2013

Elshark- 30/30

awy

2018 surgeries

\section{$\mathrm{y}$}

EA

(n)

Total knee

replacem-ent surgery

Cesarean

section

\begin{abstract}
bupivacaine
$0.5 \%+50 \mathrm{mg}$ magnesium sulphate (MgSO4)in $10 \mathrm{ml}$ as an initial bolus dose followed by infusion of $10 \mathrm{mg} / \mathrm{h}(2 \mathrm{ml} / \mathrm{h})$ during the surgery.
\end{abstract}

Bupivacaine 0.5

\%+50 mg magnesium sulphate $(\mathrm{MgSO} 4)$ in $10 \mathrm{ml}$ as an followed by infusion of 10 $\mathrm{mg} / \mathrm{h}$ (diluted in $10 \mathrm{ml}$ saline) during the surgery. initial bolus dose

N/S in
same
volume

When surgery was completed, all patients received PCEA using a PCEA device containing fentanyl $2 \mathrm{\mu g} / \mathrm{ml}$ and bupivacaine $0.03 \%(0.3 \mathrm{mg} / \mathrm{ml})$. The PCEA was programmed to deliver $2.5 \mathrm{ml} / \mathrm{h}$ infusion with a bolus dose of 1.5 $\mathrm{ml}$ on demand. The lockout interval between boluses was 6 min. If patients had inadequate analgesia, supplementary rescue analgesia with intramuscular pethidine $50 \mathrm{mg}$ was available. N/S in When surgery same was complete, volume all patients received PCEA using a PCEA device containing fentanyl $2 \mu \mathrm{g} / \mathrm{ml}$ and bupivacaine $0.08 \%$. The PCEA was programmed to administer $\mathrm{a}$ demand bolus dose of fentanyl $5 \mathrm{ml}$ with no background infusion and lockout interval $20 \mathrm{~min}$. If patients had inadequate analgesia, supplementary rescue analgesia with intramuscular pethidine $50 \mathrm{mg}$ was available. Epidural $20 \mathrm{ml} \quad \mathrm{N} / \mathrm{S}$ in If the recorded levobupivacaine hydrochloride $0.5 \%$ followed by $5 \mathrm{ml}$ of $10 \%$ preservative-free MgSO4(500 mg) prepared in two separate syringes same volume VAS was $\geq 3$, the patient was given diclofenac potassium $75 \mathrm{mg}$ oral tablets as the first rescue analgesia every $12 \mathrm{~h}$. If the VAS was still $>3$ within $30 \mathrm{~min}$, 
Ghatak $\quad 30 / 30$
2010

Gupta $\quad 30 / 30$

2013

Moham- 20/20

mad

2015
Lower

abdomina-l

and lower limb

surgeries

Total

abdomina-l

hysterectomies

EA

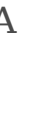

Unilatera-1

thoracic

surgery patients were

given

incremental dose

of $0.5 \mu \mathrm{g} / \mathrm{kg}$

fentanyl.

In the event of

pain, (VAS $\geq 40$ ),

both

intraoperatively

as well as

postoperatively,

a bolus of

epidural

bupivacaine

$0.25 \%(8 \mathrm{ml})$

was

administered by

the

anaesthesiologist

inside the

operation

theatre and the

nursing staff in

the recovery

room

$1 \mathrm{ml}$ of $50 \mathrm{mg} / \mathrm{ml}$

magnesium

sulphate in $10 \mathrm{ml}$

epidural

anaesthetic

solution (9 ml

$0.125 \%$

bupivacaine)

$\mathrm{N} / \mathrm{S}$ in

If patients had

same inadequate

volume analgesia (VAS

$>3$ ), epidural

fentanyl

$1 \mathrm{mcg} / \mathrm{kg}$ in $10 \mathrm{ml}$

normal saline

was

administered as

supplementary

rescue analgesic.

Epidural infusion

$\mathrm{N} / \mathrm{S}$ in

the surgery,

approximately 20

min prior to

anticipated

extubation.

Bupivacaine

$0.25 \% 8 \mathrm{ml}+$

magnesium

sulfate $50 \mathrm{mg}$ in 1

$\mathrm{ml} 0.9 \%$ saline.
Omar $\quad 50 / 50$

2018
Infraumb-ilical EA

abdomina-l

and pelvic

surgeries
$15 \mathrm{ml}$ mixture of

$14 \mathrm{ml}$

levobupivacaine

$0.5 \%+0.5 \mathrm{ml}$

MgSO410\% (50

$\mathrm{mg})+0.5 \mathrm{ml}$ of

$0.9 \mathrm{NaCl}$ in same

volume

with $5 \mathrm{ml} / \mathrm{h}$ of

$0.1 \%$

bupivacaine was

started 15 min

after the bolus

dose, and it was

continued during

the

postoperative

period via

epidural catheter

using an infusion

pump. Patients

complaining of

pain in the

postoperative

period with VAS

score $\geq 4$,

received

tramadol $50 \mathrm{mg}$

IV as rescue

analgesia and

time to the first

request for

analgesia was

noted.

$\mathrm{N} / \mathrm{S}$ in If patients had

same inadequate

volume analgesia (i.e.,: if

VAS $\geq 4$ when

measured each

hour or if patient

expressed 
epidural catheter at induction then continuous epidural infusion of this mixture by $5 \mathrm{ml} / \mathrm{h}$ till the end of the surgery

Radwan 22/22 2017

Shruthi $20 / 20$ 2016

Shahi $\quad 40 / 40$ 2014

Sun 2012
Spine

Infraumb-ilical EA surgery

Lower limb surgery surgeries
EA

Bupivacaine 0.5\% $(14 \mathrm{ml})+$ magnesium sulfate $50 \mathrm{mg}$ (in $1 \mathrm{ml}$ 0.9\% saline)

\section{i.v. drip. \\ $\mathrm{N} / \mathrm{S}$ in One gram of iv same paracetamol was volume given to the patients when VAS $>3$ and every $8 \mathrm{~h}$ thereafter. A second rescue analgesic in the form of $50 \mathrm{mg}$ iv meperidine was given to patients if VAS remained $>3$ one hour after iv paracetamol.}

N/S in Postoperative same analgesia was volume managed with epidural bolus of bupivacaine $0.125 \% 8 \mathrm{ml}$ boluses and/or Paracetamol 1 gm infusion as per discretion of treating consultants.

$\mathrm{N} / \mathrm{S}$ in same volume

In the event of pain, (VAS $\geq 40$ ), both intraoperatively as well as postoperatively, a bolus of epidural bupivacaine $0.125 \%(12 \mathrm{ml})$ was administered by the anesthesiologist inside the operation theatre and the nursing staff in the recovery room.

Cesarean CSEA After delivery of section the fetus, received $0.1 \%$ bupivacaine $10 \mathrm{~mL}$ and $\mathrm{Mg}$ $500 \mathrm{mg}$.
Nothing Postoperatively, oral diclofenac $12.5 \mathrm{mg}$ was given for rescue analgesia whenever the


CSEA:combines spinal epidural anesthesia; EA:epidural anesthesia; GA:general anesthesia; N/S:normal saline. VAS:Visual analogue scale.

\section{Figures}

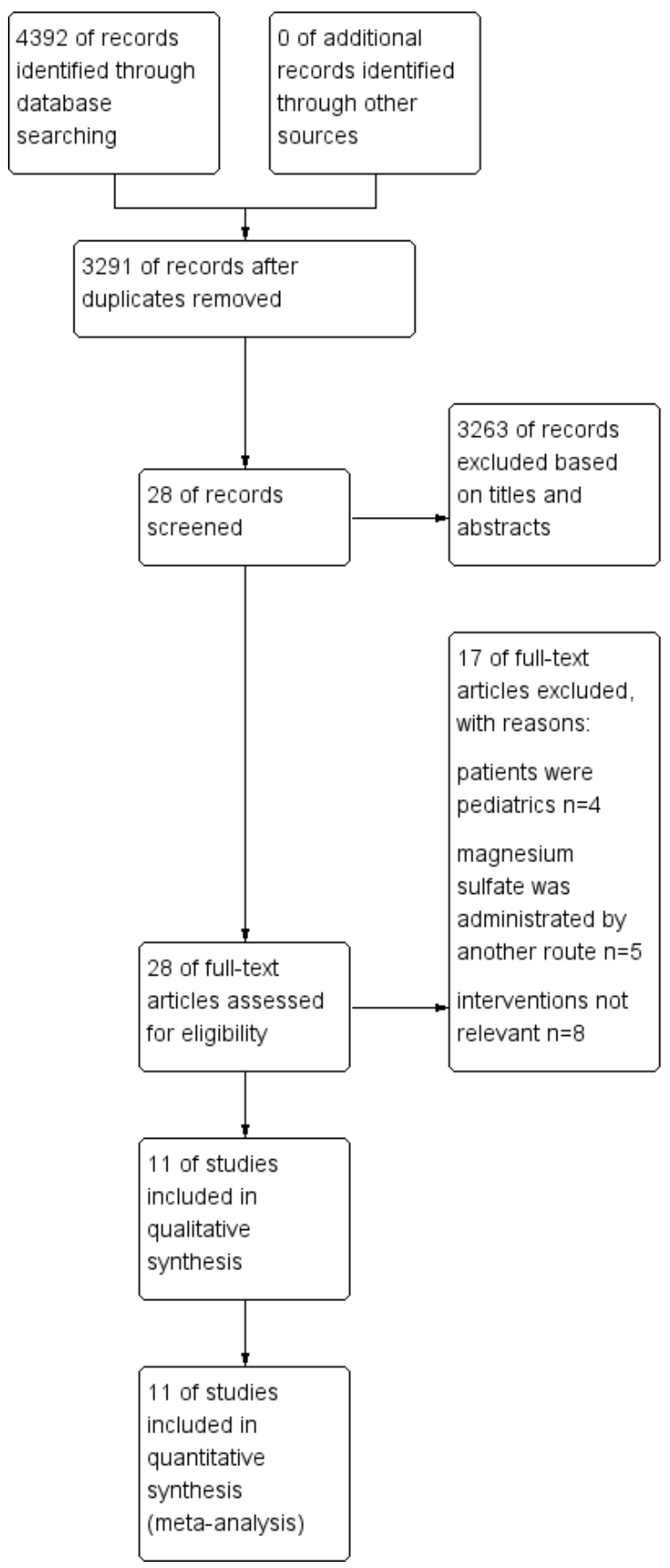

Figure 1

The details of retrieval. 


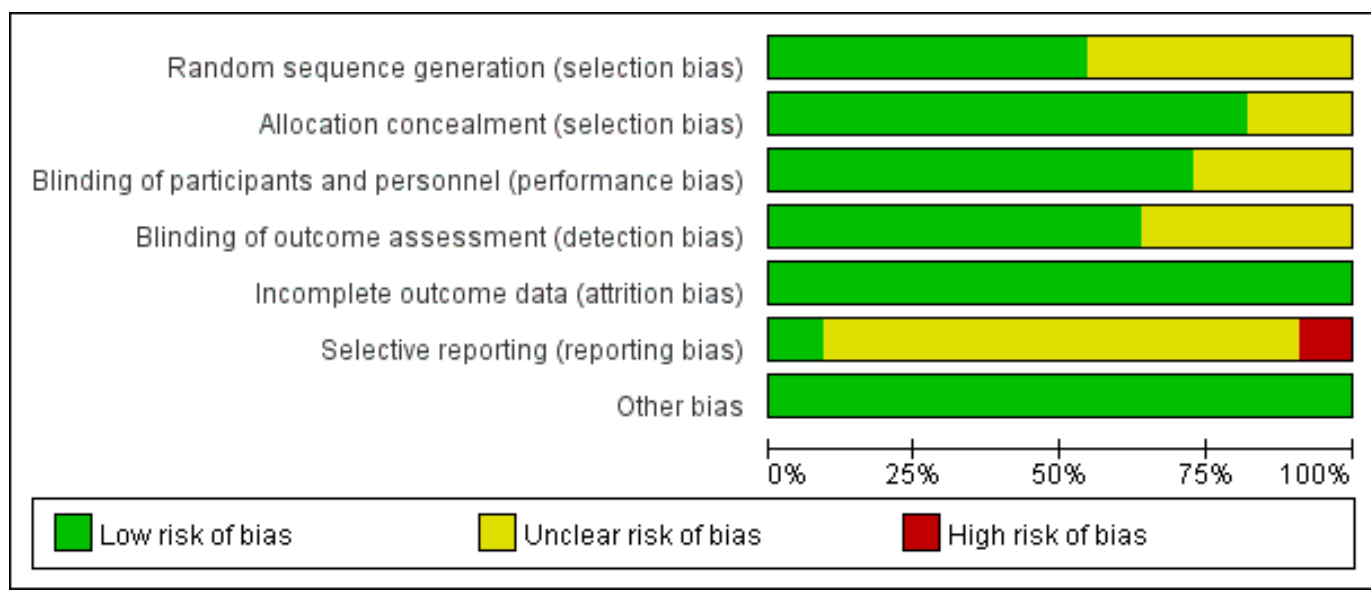

Figure 2

Risk of bias graph. Review authors' judgements about each risk of bias item presented as percentages across all included studies.

\begin{tabular}{|c|c|c|c|c|c|c|c|}
\hline & 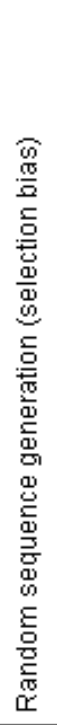 & 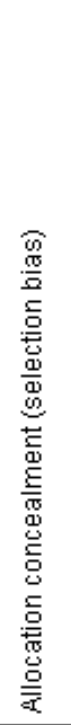 & 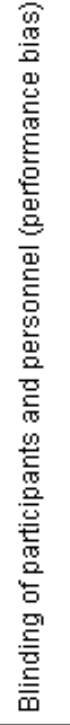 & 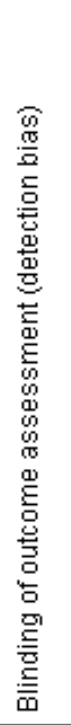 & 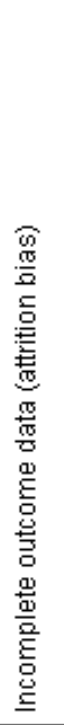 & 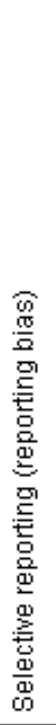 & 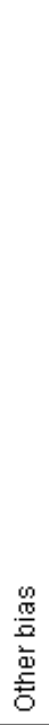 \\
\hline Aly 2011 & $?$ & + & + & + & $\odot$ & $\theta$ & + \\
\hline Daabiss 2013 & $?$ & + & + & + & $\odot$ & $?$ & + \\
\hline Elsharkawy 2018 & $?$ & + & + & + & $\odot$ & $\odot$ & + \\
\hline Ghatak 2010 & $\odot$ & $\odot$ & $\odot$ & $\odot$ & $\odot$ & $?$ & + \\
\hline Gupta 2013 & $\odot$ & $?$ & $\odot$ & $\odot$ & $\odot$ & $?$ & $\odot$ \\
\hline Mohammad 2015 & $?$ & + & $?$ & $?$ & $\odot$ & $?$ & + \\
\hline Omar 2018 & $\odot$ & + & $?$ & $?$ & $\odot$ & $?$ & + \\
\hline Radwan 2017 & $\odot$ & $\odot$ & $\odot$ & $?$ & $\odot$ & $?$ & $\odot$ \\
\hline Shahi 2014 & $?$ & + & $?$ & $?$ & $\odot$ & $?$ & + \\
\hline Shruthi 2016 & + & $?$ & + & 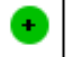 & $\odot$ & $?$ & + \\
\hline Sun 2012 & $\odot$ & + & + & + & $\odot$ & $?$ & + \\
\hline
\end{tabular}

Figure 3 
Risk of bias summary: review authors' judgements about each risk of bias item for each included study.

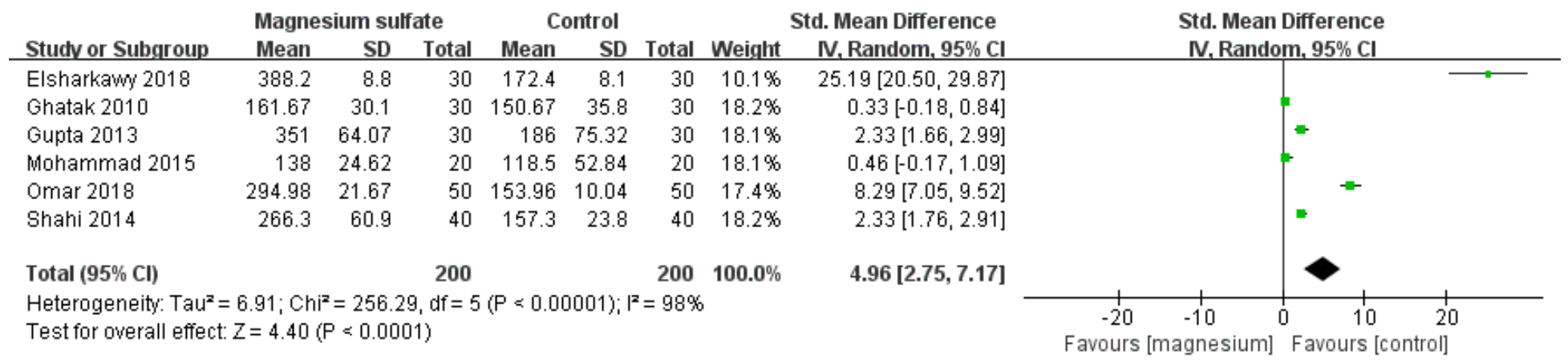

Figure 4

Forest plot of comparison: Magnesium sulfate vs Control, Outcome: the time to the first request for rescue analgesics.

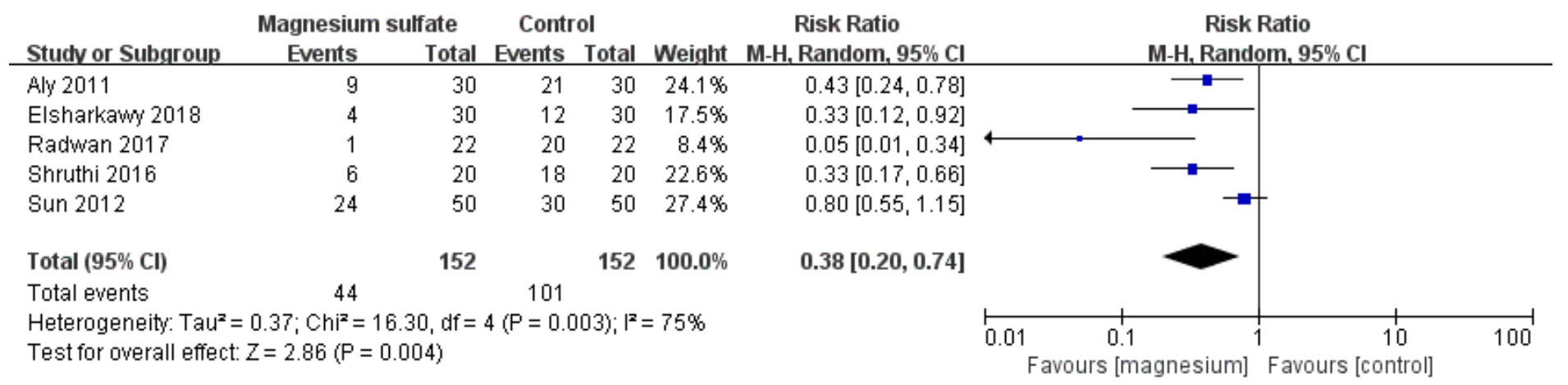

\section{Figure 5}

Forest plot of comparison: Magnesium sulfate vs Control, Outcome: the number of patients required rescue analgesics.

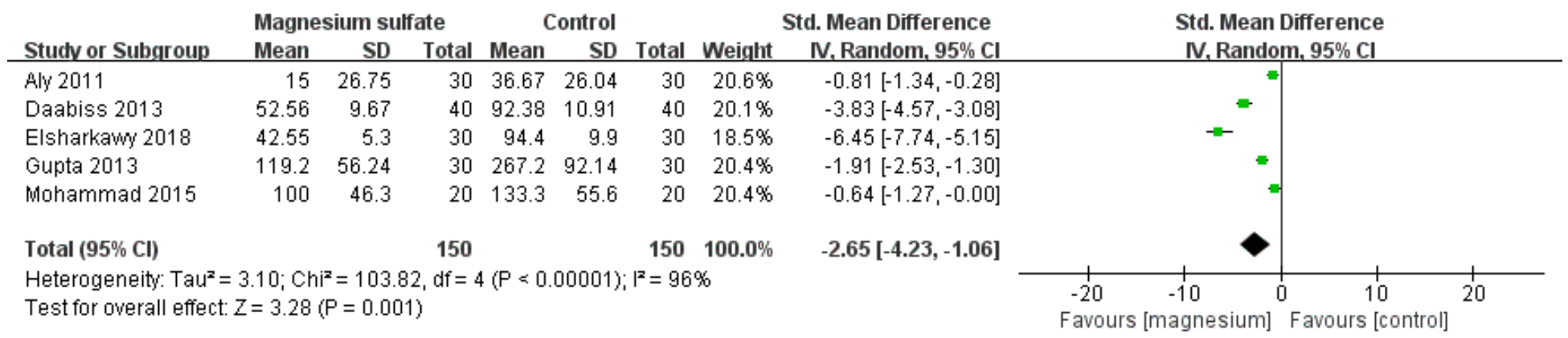

\section{Figure 6}

Forest plot of comparison: Magnesium sulfate vs Control, Outcome: the requirement for rescue analgesia.

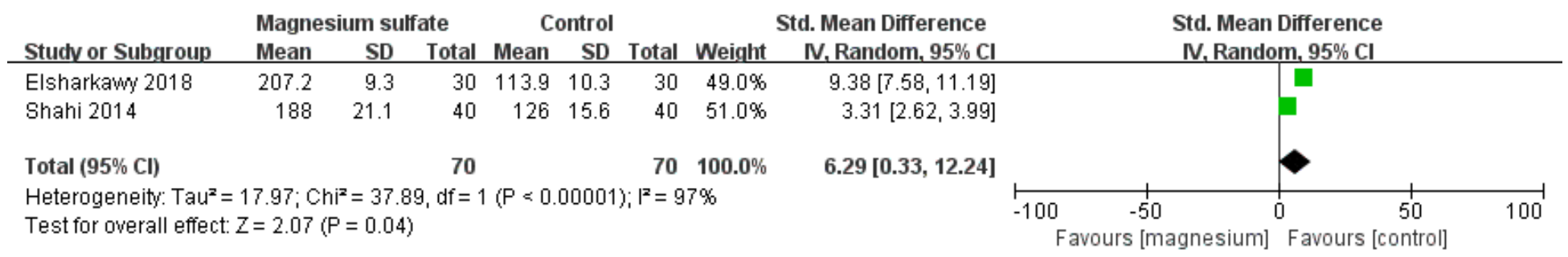

Figure 7 
Forest plot of comparison: Magnesium sulfate vs Control, Outcome: the duration of motor block.

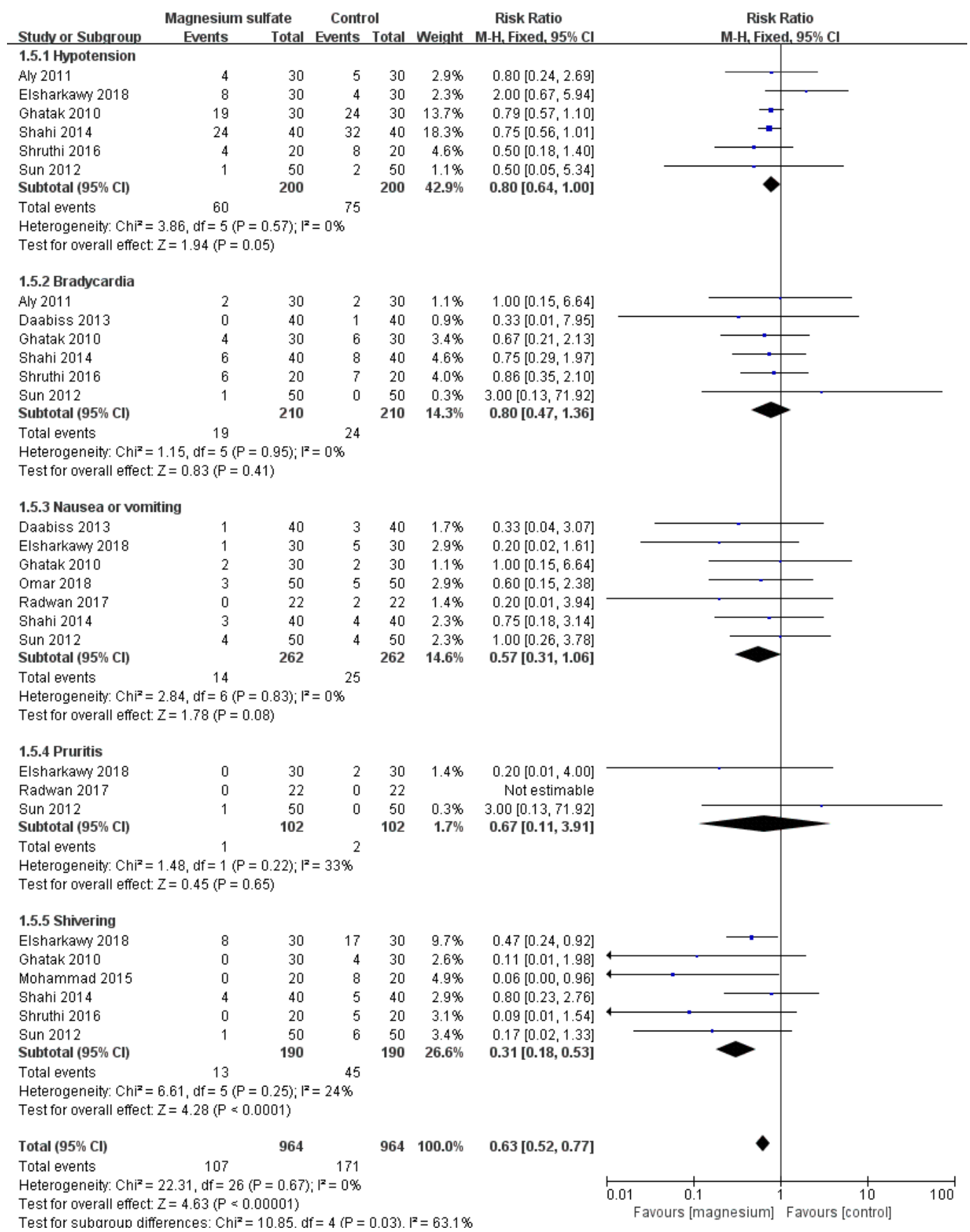

\section{Figure 8}

Forest plot of comparison: Magnesium sulfate vs Control, Outcome:the adverse events.

\section{Supplementary Files}

This is a list of supplementary files associated with this preprint. Click to download.

- Appendix1.doc

- PRISMAChecklist.doc 